\title{
Application of Thermodynamic and Kinetic Modeling to Diffusion Simulations in Nickel-Base Superalloy Systems
}

\author{
Anders Engström ${ }^{1}$, a , Johan Bratberg ${ }^{1}$, Qing Chen ${ }^{1}$, Lars Höglund ${ }^{1}$, \\ and Paul Mason ${ }^{2}$ \\ ${ }^{1}$ Thermo-Calc Software AB, Norra Stationsgatan 93 (plan 5), SE-113 64 Stockholm, SWEDEN \\ ${ }^{2}$ Thermo-Calc Software Inc., 4160 Washington Road, McMurray PA 15317, USA \\ aanders@thermocalc.se
}

\begin{abstract}
Key words: Interdiffusion, Ni-base superalloy, MCrAIY, NiAl, Coating degradation, DICTRA.
\end{abstract}
\begin{abstract}
This paper presents a brief review, followed by some new results from recent diffusion simulations in Ni-base superalloy systems, performed by means of a thermodynamic and kinetic modeling approach as taken in the commercial finite-difference code DICTRA. The DICTRA code solves the multi-component diffusion equations, combining assessed thermodynamic and kinetic data in order to determine the full composition dependent interdiffusion matrix. The link between fundamental physics based models and critically assessed data allows simulations to be performed with realistic conditions on alloys of practical importance. Emphasis in this paper is on modeling and simulation of interdiffusion occurring between NiAl coatings and Ni-base superalloy substrates. For this purpose we have used the so-called homogenization approach to diffusion in multi-phase systems, recently implemented into the DICTRA software. The simulation results have been validated against experimental data and the agreement is very satisfactory given the complexity of the problem.
\end{abstract}

\section{Introduction}

Ni-based superalloys are typically used for applications with demanding conditions and in aggressive environments. High-temperature strength, as well as resistance against creep, fatigue, oxidation, and hot corrosion is required from these alloys. For this reason these alloys are typically rather complex, e.g. containing 10 elements or more, where each element has been added for a specific purpose. Computational thermodynamics or CALPHAD [1,2] type modeling and calculations have proven very useful in improving the properties for this class of alloys by providing an accessible understanding of the influence from chemistry and processing conditions on the microstructure, see for example [3-5]. Even more detailed information on this subject has been provided by simulating the kinetics of some of the processes governing the evolution of the microstructure, e.g. using the commercial finite-difference code DICTRA [6], and also phase-field codes such as MICRESS [7]. To be more specific, DICTRA has, for example, been applied to study microsegregation related phenomena during solidification [8-10], precipitate growth/dissolution [11-14] and coarsening [15], TLP bonding [16], and interdiffusion in coating/substrate systems [1724]. In particular, the latter topic has aroused great interest from several researchers and is also the main topic of this paper.

Ni-base superalloys have excellent strength and creep resistance, but in many applications they require protective coatings that will provide formation of a continuous protective $\alpha$-alumina scale. Both overlay coatings, such as MCrAlY (where $\mathrm{M}=\mathrm{Ni}$ and/or Co), and diffusion coatings such as aluminides (e.g. NiAl) fulfill this condition and are frequently used for this purpose. In both MCrAlY and NiAl-based coatings, the Al-rich $\beta$-phase (bcc; B2) serves as an Al reservoir for the formation of a protective $\alpha$-alumina scale. During service, coating degradation occurs by two mechanisms; i) loss of Al by diffusion towards the surface of the coating to form $\alpha$-alumina and ii) interdiffusion between the coating and substrate, driven by the difference in chemical potential. Several studies have shown that the latter mechanism may contribute more to the overall Al depletion than the former one does, although repeated spallation of the $\alpha$-alumina scale may accelerate the loss of $\mathrm{Al}$ due to oxidation. It is thus most important to be able to understand and control the interdiffusion fluxes occurring between coating and substrate in order to optimize the lifetime of the coating, or ideally the performance of the component. 
An earlier study of interdiffusion in Ni-base systems was performed by Engström et al. [17] who used the so-called disperse diffusion model [25] that is implemented in DICTRA. This model is based on the assumptions that i) local equilibrium is established in each volume element at each time-step in the calculation and ii) that diffusion occurs exclusively in a continuous matrix phase. Engström et al. simulated interdiffusion in several ternary Ni-Cr-Al diffusion couples with $\gamma$-phase (fcc; A1) as the majority phase in which all diffusion was assumed to occur and with $\beta$-phase (bcc; B2) or/and $\gamma^{\prime}$-phase (fcc; $\mathrm{L1}_{2}$ ) as secondary phases. Campbell et al. [21] have used DICTRA for simulating interdiffusion in multicomponent Ni-base superalloy diffusion couples, such as IN738/Rene'-88, at a temperature where only $\gamma$-phase was present. In another, study Campbell et al. [22] used the disperse diffusion model in DICTRA for simulating interdiffusion in complex Ni-base superalloy diffusion couples, such as Rene'-95/Rene'-88, IN100/IN718, Rene'-95/IN718 and IN100/Rene'-88, containing multiphase regions, such as two-phase $(\gamma+$ MC carbide $)$ and threephase $\left(\gamma+\gamma^{\prime}+\mathrm{MC}\right.$ carbide) regions.

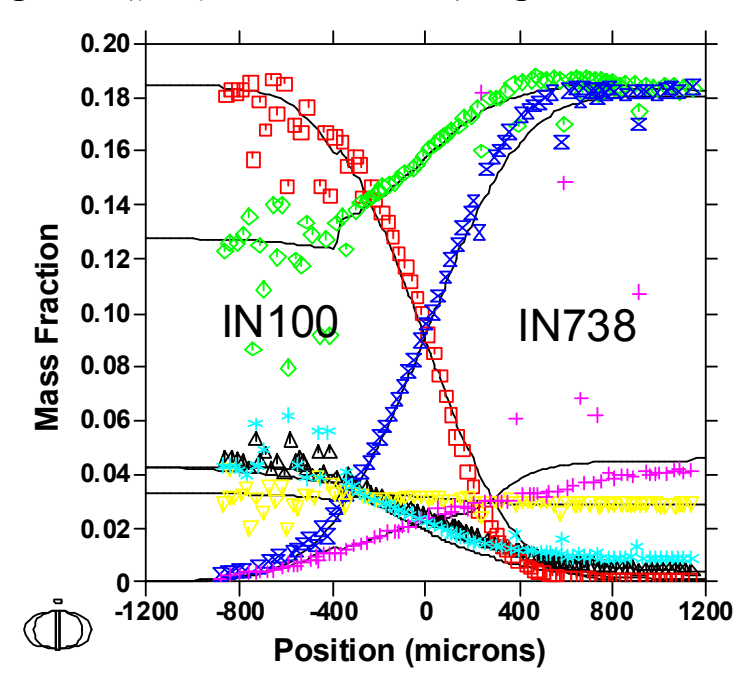

The agreement between their simulated and experimentally determined concentration profiles is excellent. This is demonstrated in Figure 1, where one of the simulations by Campbell et al. [22] has been repeated by the present authors, and where the result is compared with the experimental data from the original publication. In the simulations by Campbell at el. the amounts of secondary phases present (i.e. $\gamma^{\prime}+\mathrm{MC}$ carbide) is relatively low $(<35 \%)$ and the assumptions which underly using the disperse diffusion model are thus still valid. This is supported by the agreement with the experimentally determined profiles.

Fig. 1: Calculated concentration profiles (in mass-fraction) for $\mathrm{Al}, \mathrm{Co}, \mathrm{Cr}, \mathrm{Fe}, \mathrm{Mo}, \mathrm{Nb}$ and $\mathrm{Ti}$ in an IN100/IN738 diffusion couple after $1000 \mathrm{~h}$ of annealing at $1050^{\circ} \mathrm{C}$. Symbols are experimental data from Campbell et al [22].

Dahl et al. [23] pushed the disperse diffusion model to its limit when studying interdiffusion between an IN738 Ni-base superalloy and a Co-36.5Ni-17.5Cr-8Al-0.5Y (i.e. MCrAlY) coating at 875,925 and $950^{\circ} \mathrm{C}$, considering diffusion of $\mathrm{Al}, \mathrm{Ti}, \mathrm{Cr}, \mathrm{Co}, \mathrm{Ni}, \mathrm{Mo}, \mathrm{Ta}, \mathrm{W}$ and $\mathrm{C}$. Although the disperse diffusion model, as mentioned previously, is only considering diffusion to take place in a single continuous matrix phase, which in this case is the $\gamma$-phase, and does not consider any diffusion in either the $\beta$ - or $\gamma^{\prime}$-phase that are both present in the simulations together with the $\mathrm{M}_{23} \mathrm{C}_{6}$ carbide, Dahl et al. were still able to obtain qualitative agreement between their calculations and experimental observations. However, in the simulations by Dahl et al. the limitation of the disperse diffusion model for this type of application became evident as there was formation of a $\gamma^{\prime}$-rich layer on the immediate IN738 superalloy side of the interface due to Al interdiffusion, making the local amount of $\gamma$-phase very low (i.e. $<15 \%$ ) and thereby challenging the assumption of a continuous matrix phase as required for the disperse diffusion model. This situation is not unusual under conditions where real MCrAlY-coating/substrate systems operate, and as pointed out by Dahl et al. there is a need for a model that can take into account also diffusion taking place in the $\beta$ - and $\gamma^{\prime}$ phase. Such a model was later developed by Larsson and Engström [26] and will be briefly described in a later section of this paper. This so-called homogenization model has recently been implemented into the DICTRA software and it has been used in the current work for simulating interdiffusion between a NiAl-coating and an IN939 Ni-base superalloy substrate, as will be discussed. 


\section{Thermodynamic and kinetic data}

A critical requirement to successfully performing the interdiffusion simulations present in this paper is access to both accurate thermodynamic and kinetic CALPHAD-type databases.

There exist several thermodynamic CALPHAD-type databases for Ni-base alloys. In this work the TCNil database [27,28] has been used. The advantage of this database is the fact that the $\gamma$ - and $\gamma^{\prime}-$ phases have been modeled with a single Gibbs energy function taking into account the crystallographic relation between these two phases. Furthermore, the $\beta$-phase has been modeled with three sub-lattices (one for interstitials) which all include vacancies. This latter feature is extremely important to correctly capture the observed concentration dependency in the interdiffusion coefficients for $\beta$-NiAl.

There have been several publications on assessments of mobility data in the $\gamma$-phase for Ni-base systems [21,29,30], and a relatively comprehensive mobility database for this phase exists in MOBNi1 [31]. For the ordered phases, i.e. $\gamma^{\prime}$ - and $\beta$-phase there are less assessed data, but a good description covering the Al-Cr-Ni system has been published by Campbell [32]. For the simulations presented in this paper an extended version of the MOBNil database has been used. The extension consists of the inclusion of the assessment by Campbell for the $\gamma^{\prime}$ - and $\beta$-phase, and our own assessment of the mobilities for Co, Ti and $\mathrm{W}$ in the $\gamma^{\prime}$ - and $\beta$-phase.

\section{Simulation method}

The simulations presented in the next section have been performed using the so-called homogenization model [26], in which a diffusion flux of species $k, J_{k}$, is calculated in a latticefixed frame of reference applying Eq. 1.

$$
J_{k}=-\frac{R T}{V_{m} \Delta \widetilde{z}} \sqrt{\left[M_{k} x_{k}\right]_{n-1}^{e f f}\left[M_{k} x_{k}\right]_{n}^{\text {eff }}} 2 \sinh \left(\frac{\Delta \mu_{k}^{\text {l.eq. }}}{2 R T}\right)
$$

Where $V_{m}$ is the molar volume, which is assumed constant for substitutional species and zero for interstitial species, and $\widetilde{z}$ is a time-dependent spatial coordinate defined in the lattice-fixed frame of reference that can be related to the ordinary time-dependent spatial coordinate $z$, by keeping track of the velocity with which the lattice- and number-fixed frames of reference move with respect to each other. The number-fixed frame of reference is defined by the condition that the number of atoms on each side of a reference plane stays constant. With the above assumption for the molar volume, then the number- and volume-fixed frames of reference are identical. The fluxes are thus calculated in a lattice-fixed frame of reference, but the resulting concentration fields are mapped to number fixed frame of reference. Furthermore, $\left[M_{k} x_{k}\right]_{n-1}^{e f f}$ in Eq. 1 is a locally averaged or "effective" kinetic property on plane/source $n-1$ that is calculated by combining the product of atomic mobility for species $k$ with its fraction for all the phases present on that plane/source. Finally, $\mu_{k}^{\text {l.eq. }}$ in Eq. 1 is the chemical potential for species $k$ under local equilibrium conditions. A locally minimized Gibbs energy is assumed in this model, as is also the case for the disperse diffusion model. This means that the local phase fractions, phase compositions etc. may be obtained by performing an equilibrium calculation with the local composition. This is often, but certainly not always, a valid approximation when the local microstructural length scale is small compared to the long-range diffusion distance. It should be mentioned that the model only considers the amount or fraction of phases in each plane/source and neglects the effect of a finite rate of growth and dissolution of individual precipitates. This might be important when considering, for example, non- 
isothermal conditions where the fraction of precipitates in a plane/source might change more rapidly in the calculation than the rate of growth or dissolution of precipitates might allow for in reality. How to choose the locally averaged or "effective" kinetic properties that enter Eq. 1 is a complex problem that will not be discussed here. The interested reader is instead referred to the paper by Larsson and Engström [26]. For the calculation presented in the next section the upper Wiener bound [33] or "Rule of Mixtures" has been used, i.e.

$$
\left[M_{k} x_{k}\right]^{e f f}=\sum f^{i}\left[M_{k} x_{k}\right]^{i}
$$

where $f^{i}$ is fraction of phase $i$.The summation in Eq. 2 is taken over all phases that are to be included, i.e. in our case $\gamma, \beta$ and $\gamma^{\prime}$-phase.

\section{Simulation setup}

Perez et al. [34] have experimentally studied interdiffusion in solid-to-solid diffusion couples between single phase $\beta$-NiAl and various multiphase $\left(\gamma+\gamma^{\prime}\right)$ commercial Ni-base superalloys that were annealed at $1050^{\circ} \mathrm{C}$ for $96 \mathrm{~h}$. They measured the resulting concentration profiles by electron probe microanalysis (EPMA). In this paper a simulation will be presented comparing with one of their experimental diffusion couples formed by joining a single phase $\beta$-NiAl with an IN939 superalloy. Table 1 provides the initial concentration for IN939 reported in [34]. In our simulation we made a simplification by only including some of the elements, i.e. $\mathrm{Al}, \mathrm{Co}, \mathrm{Cr}, \mathrm{Ni}, \mathrm{Ti}$ and $\mathrm{W}$, as shown in Table 1.

Table 1: Concentration (in at\%) that was used in the simulation.

\begin{tabular}{|l|c|c|c|c|c|c|c|c|c|c|c|}
\hline Element at. \% & $\mathrm{Al}$ & $\mathrm{Co}$ & $\mathrm{Cr}$ & $\mathrm{Nb}$ & $\mathrm{Ni}$ & $\mathrm{Ta}$ & $\mathrm{Ti}$ & $\mathrm{W}$ & $\mathrm{B}$ & $\mathrm{C}$ & $\mathrm{Zr}$ \\
\hline Alloy & 51.25 & 0.001 & 0.001 & - & $\begin{array}{c}48.74 \\
6\end{array}$ & - & 0.001 & 0.001 & - & - & - \\
\hline IN939 (conc. reported in [34]) & 4.45 & 18.59 & 25.29 & 0.43 & 46.28 & 0.37 & 4.07 & 0.51 & 0.05 & 0.71 & 0.06 \\
\hline IN939 (conc. used in simulation) & 4.45 & 18.59 & 25.29 & - & 47.09 & - & 4.07 & 0.51 & - & - & - \\
\hline
\end{tabular}

As well as entering the initial (average) concentration profiles in our setup, we also provide the temperature $\left(1050^{\circ} \mathrm{C}\right)$ and simulation time $(96 \mathrm{~h})$. Furthermore, we specify the phases that we wish to include, i.e. in this case $\beta$-, $\gamma$-, $\gamma^{\prime}$ - and $\sigma$-phase. Thermodynamic and kinetic data are derived from the databases as described earlier. As already mentioned, we have for this work chosen to calculate the locally averaged or "effective" kinetic property applying the upper Wiener bound, i.e. Eq. 2. However, the $\sigma$-phase was excluded from the summation in Eq. 2 (or alternatively the mobilities in the $\sigma$-phase were approximated to be zero) due to lack of mobility data.

\section{Simulation result}

The multicomponent nature of the diffusion couple studied makes the resulting concentration profiles very complex and very different from profiles that would result from using an errorfunction for example. Even so, the agreement obtained is very satisfactory as evident from Figure 2 that shows calculated overall concentration profiles in comparison with experimental data for all the elements included in the simulation. 

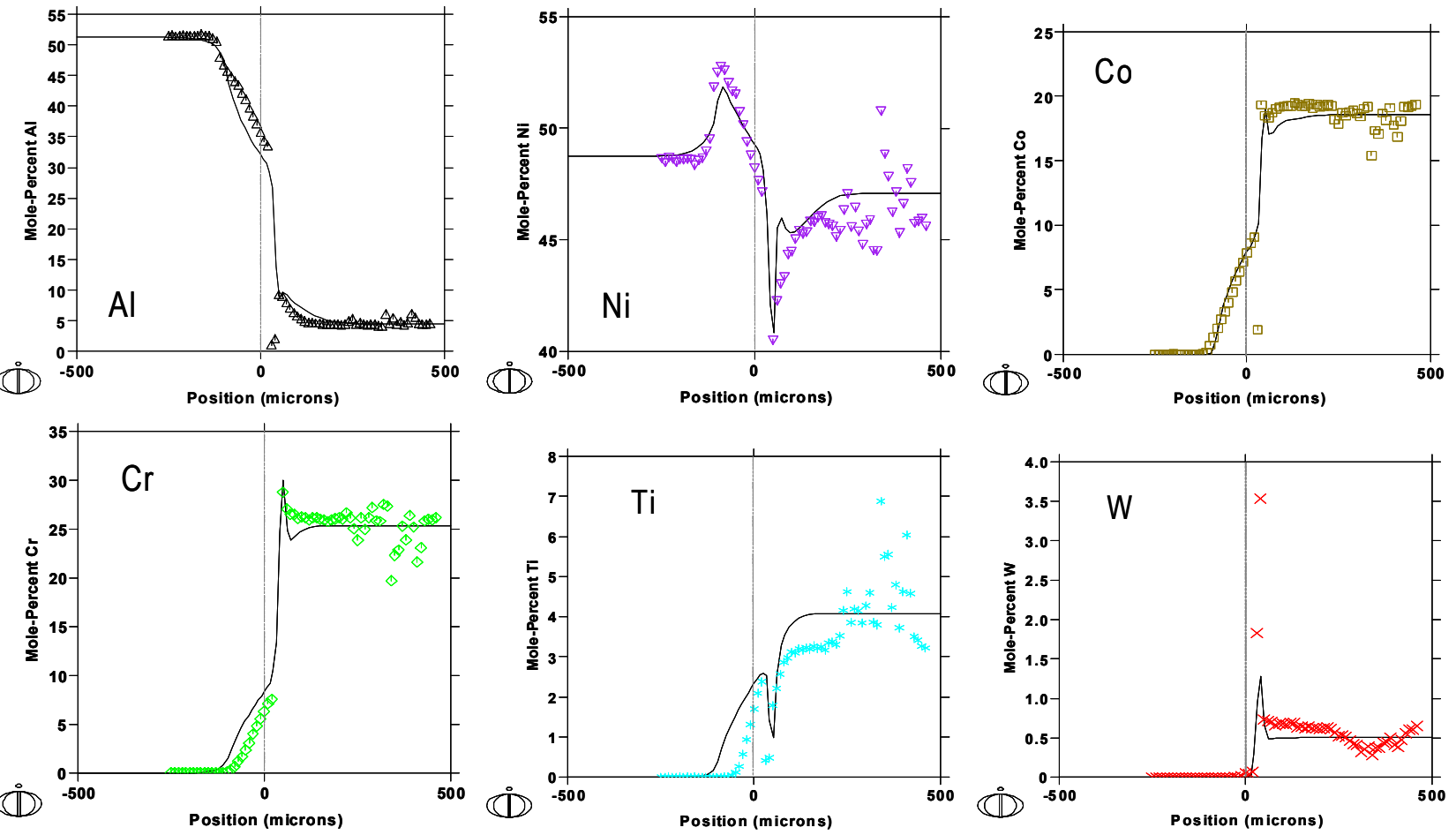

Fig. 2: Overall concentration profiles (at.\%) for $\mathrm{Al}, \mathrm{Ni}, \mathrm{Co}, \mathrm{Cr}$, Ti and $\mathrm{W}$, obtained from a simulated annealing at $1050^{\circ} \mathrm{C}$ during $96 \mathrm{~h}$ in a $\beta$-NiAl/IN939 diffusion couple. Solid lines are calculated. Symbols are experimental data from [34]. Dashed vertical line indicates the position of the initial interface.
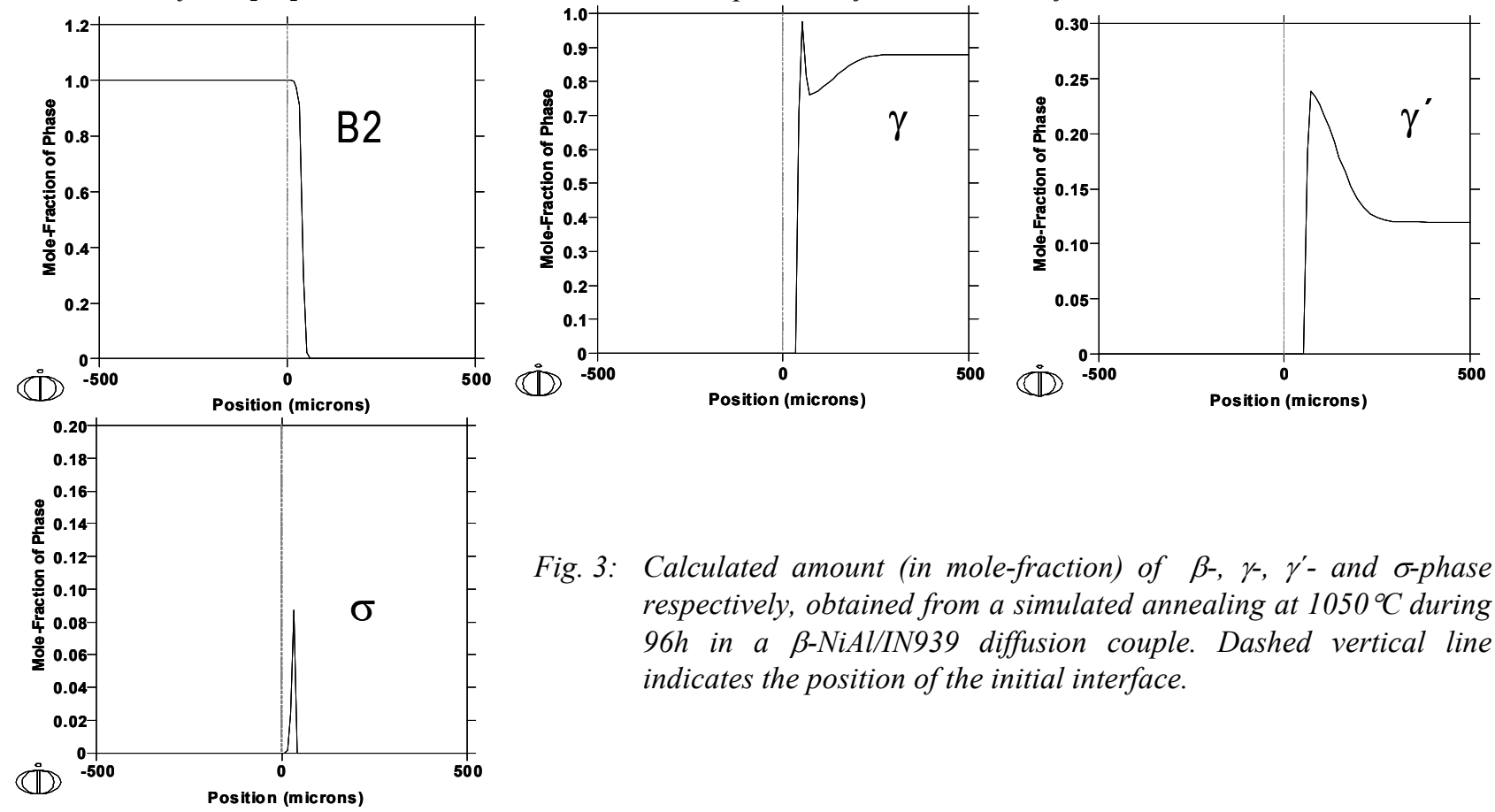

Fig. 3: Calculated amount (in mole-fraction) of $\beta$-, $\gamma$-, $\gamma^{\prime}$ - and $\sigma$-phase respectively, obtained from a simulated annealing at $1050{ }^{\circ} \mathrm{C}$ during $96 h$ in a $\beta$-NiAl/IN939 diffusion couple. Dashed vertical line indicates the position of the initial interface.

Both calculated and measured concentration profiles show for most elements either a peak or a trough immediately on the right to the initial interface, which in Figure 2 is indicated by a dashed vertical line. The reason for this may be understood by plotting the amount of respective phase predicted in the simulation. This is done in Figure 3, which shows the predicted amounts of $\beta-, \gamma-$, $\gamma^{\prime}$ - and $\sigma$-phase vs. distance though the $\beta$-NiAl / IN939 diffusion couple under consideration. As seen in Figure 3 the single $\beta$-phase region extends into the IN939 superalloy, whereas the $\gamma+\gamma^{\prime}$ two-phase region in IN939 is receding. From Figure 3 it is also evident that the simulation predicts the formation of $\sigma$-phase close to the original interface. The formation of many small precipitates rich in refractory elements was reported in [34] and the peak in the experimentally determined W concentration profile is further evidence that TCP phase, such as $\sigma$-phase, should indeed be expected close to the original interface. 


\section{References}

[1] L. Kaufman, H. Bernstain, Computer Calculation of Phase Diagrams --with Special Reference to Refractory Metals, Academic Press, Inc., New York, 1970.

[2] N. Saunders, A.P. Miodownik, CALPHAD, Calculation of Phase Diagrams, a Comprehensive Guide, 1998.

[3] H. Ohtani, K. Ishida, Thermochimica Acta 314 (1998) 69-77.

[4] N. Saunders, M. Fahrmann, C.J. Small, i:, Ninth International Symposium on Superalloys, Seven Springs, PA; USA, 2000, ss. 803-811.

[5] J. Zhao, M.F. Henry, Advanced Engineering Materials 4 (2002) 501-507.

[6] J. Andersson, T. Helander, L. Höglund, P. Shi, B. Sundman, Calphad 26 (2002) 273-312.

[7] S.G. Fries, B. Boettger, J. Eiken, I. Steinbach, Int. J. Mat. Res. (formerly Z. Metallkd.) 100 (2009) 128-134.

[8] B. Meurer, P.J. Spencer, D. Neuschutz, Zeitschrift Für Metallkunde 94 (2003) 139-143.

[9] C. Walter, B. Hallstedt, N. Warnken, Materials Science and Engineering A 397 (2005) 385390.

[10] F. Tancret, Computational Materials Science 41 (2007) 13-19.

[11] G. Bao, K. Shinozaki, M. Inkyo, T. Miyoshi, M. Yamamoto, Y. Mahara, H. Watanabe, Journal of Alloys and Compounds 419 (2006) 118-125.

[12] P. Turchi, L. Kaufman, Z. Liu, Calphad 31 (2007) 237-248.

[13] M. Miller, S. Babu, J. Vitek, Intermetallics 15 757-766.

[14] G. Bao, M. Yamamoto, K. Shinozaki, Journal of Materials Processing Technology 209 (2009) 416-425.

[15] Å. Gustafson, L. Höglund, J. Ågren, i:, Advanced Heat Resistant Steel for Power Generation; San Sebastian, San Sebatian, Spain, 1998, ss. 270-276.

[16] C. Campbell, W. Boettinger, Metallurgical and Materials Transactions A 31 (2000) 28352847.

[17] A. Engstrom, J. Morral, J. Agren, Acta Materialia 45 (1997) 1189-1199.

[18] B. Baufeld, M. Bartsch, P. Broz, M. Schmücker, Materials Science and Engineering A 384 (2004) 162-171.

[19] H. Chen, Z. Jin, C. Liu, K. Zhou, Journal of Thermal Spray Technology 13 (2004) 515-520.

[20] T. Gómez-Acebo, B. Navarcorena, F. Castro, Journal of Phase Equilibria and Diffusion 25 (2004) 237-251.

[21] C. Campbell, J. Zhao, M. Henry, Journal of Phase Equilibria and Diffusion 25 (2004) 6-15.

[22] C. Campbell, J. Zhao, M. Henry, Materials Science and Engineering: A 407 (2005) 135-146.

[23] K. Dahl, J. Hald, A. Horsewell, Defect and Diffusion Forum 258-260 (2006) 73-78.

[24] A. Jung, A. Schnell, International Journal of Fatigue 30 (2008) 286-291.

[25] A. Engström, L. Höglund, J. Ågren, Metallurgical and Materials Transactions A 25 (1994) 1127-1134.

[26] H. Larsson, A. Engstrom, Acta Materialia 54 (2006) 2431-2439.

[27] N. Dupin, B. Sundman, Scandinavian Journal of Metallurgy 30 (2001) 184-192.

[28] J. Bratberg, TCNi1, Thermo-Calc Software AB, 2008.

[29] A. Engström, J. Ågren, Zeitschrift Für Metallkunde 87 (1996) 92-97.

[30] C. Campbell, B. Boettinger, U. Kattner, Acta Materialia 50 (2002) 775-792.

[31] A. Engström, MOBNi1, Thermo-Calc Software AB, 2006.

[32] C. Campbell, Acta Materialia 56 (2008) 4277-4290.

[33] O. Wiener, Abh. Mat -Phy Kön Sächs Ges Wis 32 (1912) 509.

[34] E. Perez, T. Patterson, Y. Sohn, Journal of Phase Equilibria and Diffusion 27 (2006) 659-664. 